\title{
Correlación de la desviación de la línea media por ultrasonido transcraneal y tomografía axial computarizada
}

\author{
Edgar Manuel Gómez Martínez, ${ }^{*}$ Felipe De Jesús Montelongo, ${ }^{*, \neq}$ Jorge Arturo Nava López, ${ }^{\ddagger}$ Aurea Carmona Domínguez ${ }^{\ddagger}$
}

\section{RESUMEN}

Introducción: La ultrasonografía realizada por especialistas no radiólogos es una herramienta que contribuye al diagnóstico y monitoreo de los pacientes neurocríticos, además es económica, precisa, no invasiva y puede efectuarse a la cabecera del paciente, considerando que la gran mayoría de los pacientes neurocríticos se encuentran en inestabilidad hemodinámica. El ultrasonido se define como una serie de ondas mecánicas, generalmente longitudinales, originadas por la vibración de un cuerpo elástico (cristal piezoeléctrico) y propagadas por un medio material (tejidos corporales), cuya frecuencia supera a la del sonido audible por el humano: 20,000 ciclos/segundo o 20 kilohercios $(20 \mathrm{KHz})$. Algunos de los parámetros que se utilizan a menudo en ultrasonido son: frecuencia, velocidad de propagación, interacción del ultrasonido con los tejidos, ángulo de incidencia, atenuación y frecuencia de repetición de pulsos. Objetivo: Correlacionar la medición de la desviación de la línea media cerebral por ultrasonido (USG), con el "estándar de oro», la tomografía axial computarizada (TAC).

Material y métodos: Se realizó un estudio observacional, prospectivo, longitudinal, comparativo y analítico. Se obtuvo la medición en 22 pacientes de la desviación de la línea media cerebral por TAC y USG con diferentes patologías neurológicas. Resultados: Se obtuvo una medición media de $6.81 \mathrm{~mm}$ de desviación de la línea media cerebral de pacientes a quienes se les midió por técnica de ultrasonido y una media de $6.63 \mathrm{~mm}$ de pacientes medidos por tomografía axial computarizada de cráneo. Se correlacionó por Pearson, obteniendo una correlación de 0.774 (95\% IC), lo cual es significativo.

Discusión: Se propone la medición de la desviación de la línea media cerebral por ultrasonido transcraneal como método rápido a la cabecera del paciente, la cual genera bajos costos y puede sustituir la falta de tomografía. Puede incluirse como una medida rápida para decidir manejo quirúrgico si existiese efecto de masa, cráneo hipertensivo o focalización neurológica cuando no se cuente con tomografía. La implicación contraria a esta acepción es que el personal debe tener entrenamiento previo para realizar ultrasonido transcraneal, ya que es «operador» dependiente.

Conclusión: La medición de la línea media por ultrasonido y tomografía de cráneo se correlacionan de forma significativa, por lo que el uso de ultrasonido transcraneal puede ser un instrumento de valoración en caso de no contar con tomógrafo, aunque se requieren estudios más amplios para ser concluyentes. Palabras clave: Desviación de la línea media, tomografía axial computarizada, ultrasonido transcraneal.

\section{SUMMARY}

Background: Ultrasonography performed by non-radiological specialists is a tool that contributes to the diagnosis and monitoring of neurocritical patients, as well as being an economic, precise, non-invasive tool that can be performed at the head of the patient, considering that the vast majority of neurocritical patients are in hemodynamic instability. Ultrasound is defined as a series of mechanical waves, usually longitudinal, originated by the vibration of an elastic body (piezoelectric crystal) and propagated by a material medium (body tissues), whose frequency exceeds that of the audible sound by the human:20,000 cycles/ second or 20 kilohertz $(20 \mathrm{KHz})$. Some of the parameters that are often used in ultrasound are: frequency, velocity of propagation, interaction of ultrasound with tissues, angle of incidence, attenuation and frequency of repetition of pulses. Objective: To correlate, the measurement of the deviation of the cerebral midline by ultrasound (USG), with the "standard gold", computed axial tomography (CT).

Material and methods: An observational, prospective, longitudinal, comparative, analytical study was performed. The measurement was obtained in 22 patients of the mean deviation of the cerebral line by CAT and USG with different neurological pathologies.

\footnotetext{
* Medicina del Enfermo en Estado Crítico.

‡ Unidad de Cuidados Intensivos.
}

Hospital General Ecatepec Las Américas. ISEM. Estado de México.

Recepción: 01/09/2017. Aceptación: 13/07/2018.

Este artículo puede ser consultado en versión completa en http://www.medigraphic.com/medicinacritica
Results: A mean of $6.81 \mathrm{~mm}$ of mean deviation of the cerebral midline was obtained for patients who were measured by ultrasound technique and mean of $6.63 \mathrm{~mm}$ for patients measured by computerized axial computed tomography. It was correlated by Pearson obtaining a correlation of $0.774(95 \% \mathrm{Cl})$ which is significant.

Discussion: We propose the measurement of the deviation of the cerebral midline by transcranial ultrasound as a rapid method, at the head of the patient, which generates few costs and can replace the lack of a CT. It can be included as a quick measure to decide on surgical management if there is mass effect, hypertensive skull or neurological targeting and we do not have available tomography. The implication contrary to this meaning is that there must be a previous training of the personnel for the realization of transcranial ultrasound since it is a "dependent» operator.

Conclusion: The measurement of the midline by ultrasound and cranial tomography are significantly correlated, so the use of transcranial ultrasound can be an instrument of assessment in case of not having a tomograph, although a larger number is required of studies to be conclusive.

Key words: Midline deviation, computed tomography, transcranial ultrasound.

\section{RESUMO}

Introdução: A ultrassonografia realizada por especialistas que não são radiologistas é uma ferramenta que contribui para o diagnóstico e acompanhamento de pacientes neurocríticos, além de ser uma ferramenta não invasiva, de baixo custo e precisa, que pode ser realizada à beira do leito, considerando que a grande maioria de pacientes neurocríticos estão em instabilidade hemodinâmica. O ultrasom é definido como uma série de ondas mecânicas, geralmente longitudinais, originadas pela vibração de um corpo elástico (cristal piezoelétrico) e propagadas por um meio material (tecidos corporais), cuja frequência excede a do som audível pelo humano: 20.000 ciclos/segundo ou 20 quilohertz (20 KHz). Alguns dos parâmetros mais utilizados na ultrassonografia são: freqüência, velocidade de propagação, interação do ultrassom com os tecidos, ângulo de incidência, atenuação e frequência de repetição de pulsos.

Objetivo: Correlacionar a medida do desvio da linha média cerebral por ultrassonografia (USG) com o "padrão ouro», a tomografia axial computadorizada (TAC).

Material e métodos: Realizou-se um estudo observacional, prospectivo, longitudinal, comparativo, analítico. A medida foi obtida em 22 pacientes com o desvio médio da linha cerebral por TAC e USG com diferentes patologias neurológicas.

Resultados: Obteve-se uma medida média de $6.81 \mathrm{~mm}$ de desvio da linha média cerebral, para pacientes que foram medidos pela técnica de Ultrassonografia e média de $6.63 \mathrm{~mm}$ para pacientes medidos por Tomografia Axial Computadorizada de crânio. Foi correlacionado por Pearson obtendo uma correlação de 0.774 (IC 95\%) que é significativa.

Discussão: Propomos a medida do desvio da linha média cerebral pela ultra-sonografia transcraniana como método rápido, à beira do leito, o que gera poucos custos e pode substituir a falta de uma tomografia. Ele pode ser incluído como uma medida rápida para decidir o tratamento cirúrgico se houver um efeito de massa, um crânio hipertensivo ou uma focalização neurológica e não tivermos disponibilidade de tomografia. A implicação contrária a esse significado é que deve haver um treinamento prévio do pessoal para o ultrassom transcraniano, uma vez que ele é dependente do "operador».

Conclusões: A mensuração da linha média pela ultrassonografia e pela tomografia craniana são significativamente correlacionadas, de modo que o uso da ultrassonografia transcraniana pode ser uma ferramenta de valoração no caso de não possuir um tomógrafo, embora seja necessário um número maior de estudos para ser conclusivo.

Palavras-chave: Desvio da linha media, tomografia axial computadorizada, ultrassonografia transcraniana.

\section{INTRODUCCIÓN}

La ultrasonografía realizada por especialistas no radiólogos es una herramienta que contribuye al diagnóstico y monitoreo de los pacientes neurocríticos, además es una herramienta económica, precisa y no invasiva, que 
puede efectuarse a la cabecera del paciente, considerando que la gran mayoría de los pacientes neurocríticos se encuentran en inestabilidad hemodinámica. ${ }^{1,2}$

El ultrasonido se define como una serie de ondas mecánicas, generalmente longitudinales, originadas por la vibración de un cuerpo elástico (cristal piezoeléctrico) y propagadas por un medio material (tejidos corporales), cuya frecuencia supera a la del sonido audible por el humano: 20,000 ciclos/segundo o 20 kilohercios (20 KHz). Algunos de los parámetros que se utilizan a menudo en ultrasonido son: frecuencia, velocidad de propagación, interacción del ultrasonido con los tejidos, ángulo de incidencia, atenuación y frecuencia de repetición de pulsos. A continuación se describen brevemente cada una de estas variables. ${ }^{3}$

\section{Sonido}

Es la sensación percibida con el órgano del oído, producida por la vibración que se propaga en un medio elástico en forma de ondas.

\section{Frecuencia}

La frecuencia de una onda de ultrasonido consiste en el número de ciclos o de cambios de presión que ocurren en un segundo. La frecuencia se cuantifica en ciclos por segundo o hercios, está determinada por la fuente emisora del sonido y por el medio a través del cual viaja. El ultrasonido es un sonido cuya frecuencia se ubica por arriba de $20 \mathrm{KHz}$. Las frecuencias que se utilizan en medicina para fines de diagnóstico clínico a menudo están comprendidas en el rango de 2-30 MHz. Las frecuencias altas $(30 \mathrm{MHz})$ se usan para estructuras superficiales, por ejemplo, para valorar la piel, ojos y estructuras vasculares por vía de cateterización. Para fines experimentales se manejan frecuencias superiores a $50-200 \mathrm{MHz} .{ }^{3-5}$

\section{Velocidad de propagación}

Es la velocidad a la que el sonido viaja a través de un tejido y se considera en promedio de $1,540 \mathrm{~m} / \mathrm{s}$ en los tejidos blandos. La velocidad de propagación del sonido varía dependiendo del tipo y características del material por el que atraviese. Los factores que determinan la velocidad del sonido a través de una sustancia son la densidad y la compresibilidad, estos dos términos se refieren a la cantidad y distancia de las moléculas, respectivamente: la velocidad es inversamente proporcional a la compresibilidad; es decir, las moléculas en los tejidos más compresibles están muy separadas, por lo que trasmiten el sonido más lentamente. Por lo tanto, los materiales con más densidad y menor compresibilidad transmitirán el sonido a mayor velocidad. Esta velocidad varía en cada tejido, por ejemplo, en la grasa las ondas sonoras se mueven lentamente, mientras que en el aire la velocidad de propagación es tan lenta que las estructuras que lo contienen no pueden evaluarse por ultrasonido.

\section{Interacción con los tejidos}

Cuando la energía acústica interactúa con los tejidos corporales, las moléculas tisulares son estimuladas y la energía se transmite de una molécula a otra adyacente.

La energía acústica se mueve a través de los tejidos mediante ondas longitudinales y las moléculas del medio de transmisión oscilan en la misma dirección. Estas ondas sonoras corresponden básicamente a la rarefacción y compresión periódica del medio en el cual se desplazan. La distancia de una compresión a la siguiente (distancia entre picos de la onda sinusal) constituye la longitud de onda, la cual se obtiene al dividir la velocidad de propagación entre la frecuencia. El número de veces que se comprime una molécula es la frecuencia ( $f$ ) y se expresa en ciclos por segundo o hercios. Cuando una onda de ultrasonido atraviesa un tejido, sucede una serie de eventos, entre ellos, la reflexión o rebote de los haces ultrasónicos hacia el transductor, llamado «eco». Una reflexión ocurre en el límite o interfase entre dos materiales y provee la evidencia de que un material es diferente a otro. Esta propiedad es conocida como impedancia acústica y es el producto de la densidad y velocidad de propagación. El contacto de dos materiales con diferente impedancia acústica da lugar a una interfase entre ellos. Así es como la impedancia $(Z)$ es igual al producto de la densidad (D) de un medio por la velocidad (V) del sonido en dicho medio: $Z=V D$. Cuando dos materiales tienen la misma impedancia acústica, este límite no produce eco. Si la diferencia en la impedancia acústica es pequeña, se producirá un eco débil. Por otro lado, si la diferencia es amplia, se producirá un eco fuerte y si es muy grande, se reflejará todo el haz del ultrasonido. En los tejidos blandos, la amplitud de un eco producido en la interfase entre dos tejidos representa un pequeño porcentaje de las amplitudes. Cuando se emplea la escala de grises, las reflexiones más intensas o ecos reflejados se observan en tono blanco (hiperecoicos) y las más débiles en diversos tonos de gris (hipoecoicos) y, cuando no hay reflexiones, en negro (anecoico).

\section{Ángulo de incidencia}

La intensidad con la que un haz de ultrasonido se refleja dependerá también del ángulo de incidencia o insonación. La reflexión es máxima cuando la onda sonora incide de forma perpendicular en la interfase entre dos 
tejidos. Si el haz ultrasónico se aleja sólo a unos cuantos grados de la perpendicular, el sonido reflejado no regresará al centro de la fuente emisora y será tan sólo detectado parcialmente, o bien no será detectado por la fuente receptora (transductor).

\section{Atenuación}

Mientras las ondas ultrasónicas se propagan a través de las diferentes interfases tisulares, la energía ultrasónica pierde potencia y su intensidad disminuye progresivamente a medida que inciden estructuras más profundas (circunstancia conocida como atenuación y puede ser secundaria a la absorción o dispersión). La absorción involucra la transformación de la energía mecánica en calor, en tanto que la dispersión consiste en la desviación de la dirección de propagación de la energía. Los líquidos se consideran como no atenuadores; el hueso es un importante atenuador mediante absorción y dispersión de la energía, mientras que el aire absorbe de forma potente y dispersa la energía en todas las direcciones.

\section{Frecuencia de repetición de pulsos}

La energía eléctrica que llega al transductor estimula los cristales piezoeléctricos ahí contenidos y éstos emiten pulsos de ultrasonidos, de tal forma que el transductor no emite ultrasonidos de forma continua, sino que genera grupos o ciclos de ultrasonidos a manera de pulsos. La frecuencia con la que el generador produce pulsos eléctricos en un segundo se llama frecuencia de repetición de pulsos, mejor conocida por sus siglas en inglés (PRF) y es igual a la frecuencia de repetición de pulsos de ultrasonidos (número de veces que los cristales del transductor son estimulados por segundo). Por lo tanto, la PRF determina el intervalo entre las dos fases: emisión y recepción de los ultrasonidos. Este intervalo debe ser el adecuado para que, de manera coordinada, un pulso de ultrasonido alcance un punto determinado en profundidad y vuelva en forma de eco al transductor antes de que se emita el siguiente pulso. EI PRF depende, entonces, de la profundidad de la imagen y suele variar entre 1,000 y $10,000 \mathrm{KHz}$. Cada uno de los pulsos recibidos y digitalizados pasan a la memoria gráfica, se ordenan, se procesan y se presentan en forma de puntos brillantes en el monitor, en éste se emiten secuencias de al menos 20 barridos tomográficos por segundo para ser visualizados en tiempo real.

\section{Resolución}

Es la habilidad de distinguir las diferentes partículas que reflejan el ultrasonido. Los diferentes tejidos que se ubican cerca proporcionan reflexiones individuales.
La resolución se refiere a la nitidez y al detalle de la imagen. En ecografía, la resolución depende de dos características inherentes a la agudeza visual: el detalle y el contraste. La resolución lineal determina qué tan lejanos se ven dos cuerpos reflejados, de tal manera que puedan discriminarse como puntos separados. La resolución de contraste determina la diferencia de amplitud que deben tener dos ecos antes de ser asignados a diferentes niveles de gris.

\section{Escala de grises}

Las estructuras corporales están formadas por distintos tejidos, lo que da lugar a múltiples interfases que originan, en imagen digital, la escala de grises. El elemento orgánico que mejor transmite los ultrasonidos es el agua, ya que produce una imagen ultrasonográfica anecoica. En general, los tejidos muy celulares son hipoecoicos gracias a su alto contenido de agua, mientras que los tejidos fibrosos son hiperecoicos debido al mayor número de interfases presentes en ellos.

\section{Transductores}

Un transductor es un dispositivo capaz de transformar o convertir un determinado tipo de energía de entrada en otro diferente a la salida. En el caso de los transductores de ultrasonido, la energía ultrasónica se genera en el transductor que contiene los cristales piezoeléctricos. Éstos poseen la capacidad de transformar la energía eléctrica en sonido y viceversa, de tal manera que el transductor o sonda actúa como emisor y receptor de ultrasonidos.

La circonita de plomo con titanio es una cerámica usada frecuentemente como cristal piezoeléctrico y constituye el alma del transductor; recientemente, se desarrollaron polímeros piezoeléctricos como polivinilideno (PVDF) y trifluoroetileno (TrFE), que han demostrado ser útiles para producir frecuencias altas (> 100 $\mathrm{MHz}$ ). Existen varios tipos de transductores que difieren tan sólo en la manera en que están dispuestos sus componentes. Los transductores sectoriales tienen una ventana pequeña, por ejemplo, para la visualización de las costillas con un ángulo de escaneo ancho; los convexos tienen un amplio campo a distancia con un tamaño de ventana adecuado y los lineales se caracterizan por tener un amplio plano de contacto, ideal para pequeñas estructuras. Estos últimos son los que se emplean con más frecuencia en la ultrasonografía musculoesquelética, se componen de un número variable de cristales piezoeléctricos (usualmente de 64 a 256) que se disponen de forma rectangular, se sitúan uno frente al otro y funcionan en grupos, de modo que al ser estimulados eléctricamente producen o emiten simultáneamente un haz ultrasónico. Los tres transductores a utilizar en en- 
fermos críticos son el lineal, de alta frecuencia (7.5-10 $\mathrm{MHz}$ ), el sectorial, de baja frecuencia (2-4 MHz), y el convexo de frecuencia media $(3.5-5 \mathrm{MHz})$.

\section{Creación de la imagen}

Las imágenes ecográficas están formadas por una matriz de elementos fotográficos. Las imágenes en escala de grises se generan por la visualización de los ecos, regresando al transductor como elementos fotográficos (pixeles). Su brillo dependerá de la intensidad del eco, que es captado por el transductor en su viaje de retorno. El transductor se coloca sobre la superficie corporal del paciente a través de una capa de gel para eliminar el aire entre las superficies (transductor-piel). Un circuito transmisor aplica un pulso eléctrico de pequeño voltaje a los electrodos del cristal piezoeléctrico. Éste empieza a vibrar y transmite un haz ultrasónico de corta duración, el cual se propaga dentro del paciente, donde es parcialmente reflejado y transmitido por los tejidos o interfases tisulares que encuentra a su paso.

La energía reflejada regresa al transductor y produce vibraciones en el cristal, las cuales son transformadas en corriente eléctrica por el cristal y después son amplificadas y procesadas para transformarse en imágenes. El circuito receptor puede determinar la amplitud de la onda sonora de retorno y el tiempo de transmisión total, ya que rastrea cuándo se transmite y cuándo retorna. Al conocer el tiempo del recorrido, es posible calcular la profundidad del tejido refractante usando la constante de 1,540 metros/segundo como velocidad del sonido. La amplitud de la onda sonora de retorno determina la gama o tonalidad de gris que deberá asignarse. Los ecos muy débiles dan una sombra cercana al negro dentro de la escala de grises, mientras que los ecos potentes dan una sombra cercana al blanco.

\section{Modalidades de la ecografía}

Existen tres modos básicos de presentar las imágenes ecográficas.

El modo A o de amplitud fue el que se empleó inicialmente para distinguir entre estructuras quísticas y sólidas y se utilizó para representar gráficamente una señal. Hoy en día, su uso es muy extendido, salvo para comprobar los parámetros técnicos viendo la amplitud a distintas profundidades.

El modo $\mathbf{M}$ se emplea para las estructuras en movimiento como el corazón; se realiza una representación gráfica de la señal, la amplitud es el eje vertical y el tiempo y la profundidad son el eje horizontal.

EI modo B es la representación pictórica de la suma de los ecos en diferentes direcciones (axial, lateral), lo que ayuda a que el equipo reconozca la posición espacial y la dirección del haz. Las señales de eco detecta- das son procesadas y transmitidas a luminosidad, dando como resultado un brillo. Las estructuras con más reflejo parecen más brillantes que las estructuras con menos reflejo. Ésta es la modalidad que se emplea en todos los equipos de ecografía en tiempo real y se trata de una imagen bidimensional estática. ${ }^{6,7}$

\section{Historia}

En 1977, Becker et al. señalaron un doble aumento en la mortalidad cuando la desviación de la línea media cerebral superó $1 \mathrm{~cm}$ (53\% frente a $25 \%)$. Recientemente, se ha demostrado que una desviación de la línea media cerebral por encima de $5 \mathrm{~mm}$ en la tomografía axial computarizada cerebral inicial predice un resultado neurológico deficiente con un valor predictivo positivo de $78 \%$, mientras que sólo $14 \%$ de los casos sin desviación de la línea media en la tomografía axial computarizada se asoció a un mal resultado. ${ }^{8}$ Se ha observado que la desviación de la línea media en la TAC se correlaciona con la puntuación de coma de Glasgow y con otros signos tomográficos de gravedad. Marshall et al. propusieron una clasificación de tomografía computarizada basada en datos (Traumatic Coma Data Bank), la cual incluye una desviación de la línea media cerebral mayor de $5 \mathrm{~mm}$ como uno de los principales criterios en la TAC para la gravedad de la lesión cerebral traumática (TBI); y un análisis multivariado de una cohorte de más de 10,000 pacientes con TBI mostró que la compresión del tercer ventrículo y una desviación de la línea media cerebral mayor de $5 \mathrm{~mm}$ fueron los principales predictores de mortalidad dentro de los primeros 15 días después de la lesión. Del mismo modo, Ropper, Pullicino et al. observaron que, tras un accidente cerebrovascular, la alteración de la consciencia era directamente proporcional a la desviación de la línea media cerebral en la TAC. ${ }^{9-12}$

Por lo tanto, la detección oportuna de la desviación de la línea media en pacientes con patología cerebral aguda es muy importante, porque permite la implementación de un plan de tratamiento adecuado (según las recomendaciones en Estados Unidos en 2006, en el caso de desviación de la línea media mayor de $5 \mathrm{~mm}$ en presencia de TBI grave se requería una evacuación quirúrgica). Sin embargo, a pesar de que la TAC de cráneo se considera el estándar de oro para diagnosticar desviación de la línea media, la ecografía del cerebro ha demostrado ser capaz de visualizar la mayoría de las estructuras intracerebrales. Esta tecnología es no invasiva, se asocia a baja exposición de radiación, puede aplicarse a la cabecera de la cama del paciente y se ha utilizado como herramienta adicional para la evaluación de pacientes con accidente cerebrovascular isquémico agudo. Seidel et al. ${ }^{13}$ describieron en 1996 un método simple para determinar la desviación de la línea media 
con sonografía. Esto pareció correlacionarse bien con los hallazgos en la TAC y ser útil como predictor de pronóstico rápido al detectar de inmediato una desviación de la línea media cerebral significativa en el ictus agudo; sin embargo, esto sólo se describió en un pequeño estudio de pacientes con TBI.

\section{Técnica de medición}

La medición de la desviación de la línea media se realiza utilizando el tercer ventrículo como estructura de referencia, ya que presenta una buena correlación con la tomografía de cráneo. La medición de la desviación de la línea media se calcula con la fórmula $(A-B) / 2$, siendo A la medición obtenida desde el término de la tabla interna e inicio de las estructuras cerebrales (borde hiperecogénico del término de la tabla interna) hasta el centro del tercer ventrículo de un lado y $B$ repitiendo el mismo procedimiento, insonando desde el lado contralateral. Una medición de la desviación de la línea media de más de $4 \mathrm{~mm}$ se considera relevante. En los casos de defectos óseos, por ejemplo, craniectomías descompresivas o fracturas desplazadas, la medición de la desviación de la línea media con este método puede ser imprecisa. En pacientes con craniectomías descompresivas se ha propuesto insonar directamente sobre el defecto óseo, observando sólo el tejido cerebral, lo que permite objetivar la desviación de la línea media, utilizando el desplazamiento del septum pellucidum en relación con la hoz hiperecogénica del cerebro, esta última es la que se utiliza como referencia de la línea media y no requiere una referencia anatómica específica. ${ }^{14,15}$

El desplazamiento de la línea media cerebral es uno de los parámetros que denota severidad en patologías neurológicas y en ocasiones define el manejo quirúrgico. Reportes de casos han mostrado que es posible visualizar la línea media en la ventana ósea a través de la escama del hueso temporal. ${ }^{16}$

Para su realización se requiere de un transductor de baja frecuencia (2-4 MHz) (sectorial) que optimiza las imágenes cuando se efectúa ultrasonografía del cerebro, las estructuras serán visualizadas en modo $B$, puede visualizar el mesencéfalo, los ventrículos laterales y la línea media; esta última se ha reportado desviada en pacientes con ataque cerebrovascular, hematomas durales y ocasionalmente hemorragias de ganglio basales.

Es importante recordar que hasta $15 \%$ de los pacientes no tienen una buena ventana ultrasonográfica, por lo que estas estructuras no serán visibles. En pacientes con craniectomía descompresiva se facilita la valoración del parénquima cerebral, puesto que no aparecen los artefactos y la sombra acústica ósea. En estos pacientes el transductor se posiciona en los sitios donde no hay tejido óseo y no requiere una referencia anatómica específica. En este grupo de pacientes se ha reportado seguimiento de tamaño de hematomas intracerebrales, monitoreo o seguimiento de la desviación de la línea media con el tratamiento instaurado. ${ }^{17-19}$

\section{MATERIAL Y MÉTODOS}

Tipo de estudio: Se realizó un estudio observacional, prospectivo, longitudinal, comparativo y analítico.

Criterios de inclusión: Pacientes de 16 a 100 años de edad. Pacientes con patología cerebral aguda ingresados en la unidad de cuidados neurocríticos. Pacientes con patología cerebral aguda postoperados de craneotomía o craniectomía descompresiva que tienen programada tomografía axial computarizada de cráneo. Pacientes con patología cerebral aguda a quienes se les realizó tomografía axial computarizada de cráneo no programada. Pacientes a quienes se les realizó ultrasonido transcraneal 20 minutos antes de la tomografía axial computarizada de cráneo.

Criterios de exclusión: Pacientes menores de 16 años que se encuentren hospitalizados en unidad de cuidados neurocríticos. Pacientes hospitalizados en la unidad de cuidados neurocríticos sin tomografía axial computarizada.

Criterios de eliminación: Pacientes que no aceptaron participar en el estudio de tomografía axial computarizada de cráneo y/o ultrasonografía transcraneal en el consentimiento informado.

Definición de variables: Línea media cerebral. Línea media de estructuras encefálicas ocupada por la cisura interhemisférica, cuerpo calloso, fórnix, tercer ventrículo, septum pellucidum y glándula pineal, una desviación mayor de $4 \mathrm{~mm}$ obtenida por ultrasonido transcraneal o mayor de $5 \mathrm{~mm}$ por tomografía axial computarizada se considera relevante (variable independiente, cuantitativa, discreta). ${ }^{20}$

Universo de trabajo: Se incluyeron 22 pacientes de la unidad de cuidados neurocríticos con diferentes patologías neurológicas. Se realizó ultrasonido transcraneal previo a tomografía de cráneo, en algunos casos en el mismo paciente posterior a craniectomía o craneotomía para comparar mediciones y definir la utilidad como método en tiempo real y repetitivo en la cama del paciente.

Tamaño de muestra: El estudio se realizó en un periodo que abarcó desde enero de 2017 hasta la fecha en pacientes de la unidad de cuidados neurocríticos.

Método: Se utilizó equipo de tomografía axial computarizada y un equipo de ultrasonido Doppler, Micromaxx, marca Sonosite, con transductor sectorial de 2-4 $\mathrm{MHz}$.

Se solicitó consentimiento informado (Anexo 1) al familiar o responsable del paciente para la realización de la tomografía axial computarizada de cráneo y ultrasonido transcraneal. 
Método tomográfico (Anexo 2): Trazar una línea desde la cresta frontal interna hasta la cresta occipital interna, definida como línea media ósea; la línea media de estructuras encefálicas ocupada por la cisura interhemisférica, cuerpo calloso, fórnix, tercer ventrículo y glándula pineal. Para establecer la magnitud de desplazamiento de la línea media, se calcula la diferencia entre la medición de la línea media ideal (normal) y el septum pellucidum.

Método ultrasonográfico: Ventana transtemporal: por encima del arco cigomático, delante del trago y detrás de la comisura lateral del ojo. Identificar tabla interna del hueso temporal ipsilateral. Identificar el término de la tabla interna (borde hiperecogénico) e inicio de las estructuras cerebrales. Identificar la estructura media, línea horizontal hiperecogénica (septum pellucidum) que divide ambos ventrículos. Identificar el tronco cerebral como referencia anatómica. Realizar la medición en ventana transtemporal izquierda y derecha, aplicar la fórmula $(A-B) / 2$. Reportar la medición en milímetros y si la desviación es hacia la izquierda o derecha. ${ }^{21,22}$

Diseño estadístico: El análisis se hizo con la prueba estadística de correlación de Pearson, se utilizó el software computacional SPSS para la realización y descripción gráfica.

\section{RESULTADOS (ANEXO 3)}

Se obtuvo una media de $6.81 \mathrm{~mm}$ de pacientes a quienes se les midió la desviación de la línea media cerebral con ultrasonido transcraneal y una media de $6.63 \mathrm{~mm}$ de pacientes con tomografía axial computarizada de cráneo. Se correlacionó por Pearson y se obtuvo como resultado 0.774 (95\% IC), lo que significa que la medición por ultrasonido de la línea media es útil en relación con la misma medida realizada por TAC para evaluar el desplazamiento de los hemisferios cerebrales.

\section{DISCUSIÓN}

Del análisis estadístico se obtuvo un resultado de 0.774 , que se traduce en una correlación positiva alta en la medición de línea media por ultrasonido en comparación con la medición por tomografía; de tal forma que proponemos la medición de la desviación de la línea media por ultrasonido transcraneal como método rápido, a la cabecera del paciente, a bajos costos y que puede sustituir la falta de una tomografía axial computarizada. Puede incluirse como una medida rápida para decidir manejo quirúrgico si existiese efecto de masa, cráneo hipertensivo o focalización neurológica y no se cuente con tomógrafo. La implicación contraria a esta acepción es que el personal debe tener entrenamiento previo para realizar el ultrasonido transcraneal, ya que es un método «operador» dependiente.

\section{CONCLUSIÓN}

La medición de la línea media por ultrasonido y tomografía de cráneo se correlacionan de forma significativa, por lo que el uso de ultrasonido transcraneal puede ser un instrumento de valoración en el paciente con patología cerebral aguda en caso de no contar con tomógrafo, aunque se requieren estudios más amplios para ser concluyentes.

\section{BIBLIOGRAFÍA}

1. Andruszkiewicz $P$, Sobczyk D. Ultrasound in critical care. Anaesthesiol Intensive Ther. 2013;45(3):177-181.

2. Rincon F. Bedside transcranial sonography: A promising tool for the neurointensivist. Crit Care Med. 2012;40(6):1969-1970.

3. Caricato A, Mignani V, Bocci MG, Pennisi MA, Sandroni C, Tersali $A$, et al. Usefulness of transcranial echography in patients with decompressive craniectomy: a comparison with computed tomography scan. Crit Care Med. 2012;40(6):1745-1752.

4. Berg D, Godau J, Walter U. Transcranial sonography in movement disorders. Lancet Neurol. 2008;7(11):1044-1055.

5. Lucas Ochoa-Péreza, ${ }^{*}$ y Alejandro Cardozo-Ocampob. Aplicaciones de la ultrasonografía en el sistema nervioso central para neuroanestesia y cuidado neurocrítico. Colombian Journal of Anesthesiology. 2015;43(4):314-320.

6. Motuel J, Biette I, Srairi M, Mrozek S, Kurrek MM, Chaynes P, et al. Assessment of brain midline shift using sonography in neurosurgical ICU patients. Crit Care. 2014;18(6):676.

7. Eisenberg HM, Gary HE Jr, Aldrich EF, Saydjari C, Turner B, Foulkes MA, et al. Initial CT findings in 753 patients with severe head injury. A report from the NIH traumatic coma data bank. $J$ Neurosurg. 1990;73(5):688-698.

8. Fearnside MR, Cook RJ, McDougall P, McNeil RJ. The Westmead head injury project outcome in severe head injury. a comparative analysis of pre-hospital, clinical and CT variables. Br J Neurosurg. 1993;7(3):267-279.

9. Yanaka K, Kamezaki T, Yamada T, Takano S, Meguro K, Nose $\mathrm{T}$. Acute subdural hematoma-prediction of outcome with a linear discriminant function. Neurol Med Chir (Tokyo). 1993;33(8):552-558.

10. Kotwica Z, Brzezinski J. Acute subdural haematoma in adults: an analysis of outcome in comatose patients. Acta Neurochir (Wien). 1993;121(3-4):95-99.

11. Foundation TBT. The American Association of Neurological Surgeons. The Joint Section on Neurotrauma and Critical Care. Computed tomography scan features. J Neurotrauma. 2000;17(6-7):597-627.

12. Marshall LF, Marshall SB, Klauber MR, Van Berkum Clark $M$, Eisenberg $\mathrm{H}$, Jane JA, et al. A new classification of head injury based on computerized tomography. J Neurosurg. 1991;75:S14-S20.

13. Maas Al, Hukkelhoven CW, Marshall LF, Steyerberg EW. Prediction of outcome in traumatic brain injury with computed tomographic characteristics: a comparison between the computed tomographic classification and combinations of computed tomographic predictors. Neurosurgery. 2005;57:11731182.

14. Bullock MR, Chesnut R, Ghajar J, Gordon D, Hartl R, Newell DW, et al. Surgical management of acute subdural hematomas. Neurosurgery. 2006;58(Suppl. 3):S16-S24.

15. Andrews PJ, Piper IR, Dearden NM, Miller JD. Secondary insults during intrahospital transport of head-injured patients. Lancet. 1990;335(8685):327-330.

16. Kaups KL, Davis JW, Parks SN. Routinely repeated computed tomography after blunt head trauma: does it benefit patients? $J$ Trauma. 2004;56(3):475-480.

17. Bogdahn U, Becker G, Winkler J, Greiner K, Perez J, Meurers B. Transcranial color-coded real-time sonography in adults. Stroke. 1990;21(12):1680-1688. 
18. Seidel G, Gerriets T, Kaps M, Missler U. Dislocation of the third ventricle due to space-occupying stroke evaluated by transcranial duplex sonography. J Neuroimaging. 1996;6(4):227-230.

19. Stolz E, Gerriets T, Fiss I, Babacan SS, Seidel G, Kaps M. Comparison of transcranial color-coded duplex sonography and cranial CT measurements for determining third ventricle midline shift in space-occupying stroke. AJNR Am J Neuroradiol. 1999;20(8):1567-1571.

20. Gerriets T, Stolz E, Modrau B, Fiss I, Seidel G, Kaps M. Sonographic monitoring of midline shift in hemispheric infarctions. Neurology. 1999;52(1):45-49.

21. Gerriets T, Stolz E, Konig S, Babacan S, Fiss I, Jauss M, et al. Sonographicmonitoring of midline shift in space-occupying stroke: an early outcome predictor. Stroke. 2001;32(2):442-447.

22. Llompart PJ, Abadal CJ, Palmer SM, Perez BJ, Casares VM, Homar RJ, et al. Monitoring midline shift by transcranial color coded sonography in traumatic brain injury. A comparison with cranial computerized tomography. Intensive Care Med. 2004;30(8):1672-1675.

Correspondencia:

Edgar Manuel Gómez Martínez

Prolongación de Profirió Díaz Núm. 124,

Col. Miguel Alemán,

68120, Oaxaca de Juárez, Oaxaca,

Tel: (951) 41248

Cel: (044) 9512687990

E-mail: umqedgar@hotmail.com

\section{ANEXO 1 \\ HOJA DE CONSENTIMIENTO INFORMADO}

Unidad de Cuidados Neurocríticos

Lugar:

Fecha:

Hora:

Nombre del paciente: Edad:

Nombre del familiar responsable:

Parentesco:

Por medio del presente documento se me ha informado ampliamente el diagnóstico de mi paciente, así como de la realización de tomografía axial computarizada de cráneo y ultrasonido transcraneal, procedimientos no invasivos, por lo que firmo de conformidad la aceptación para la realización de los estudios de gabinete. 


\section{ANEXO 2}

\section{HOJA DE RECOLECCIÓN DE DATOS}

Nombre:

Edad:

Sexo: Número de expediente:

Fecha:

\section{Método tomográfico}

- Trazar una línea desde la cresta frontal interna hasta la cresta occipital interna, definida como línea media ósea.

- Para establecer la magnitud de desplazamiento de la línea media, se calcula la diferencia entre la medición de la línea media ideal (normal) y el septum pellucidum.

\section{Método ultrasonográfico}

$\begin{array}{ll}\text { Ventana transtemporal } & \text { Ventana transtemporal } \\ \text { Derecha } & \text { Izquierda }\end{array}$

- Ventana transtemporal: por encima del arco cigomático, delante del trago y detrás de la comisura lateral del ojo.

- Identificar tabla interna del hueso temporal ipsilateral.

- Identificar el término de la tabla interna (borde hiperecogénico e inicio de las estructuras cerebrales.

- Identificar la estructura media, línea horizontal hiperecogénica (septum pellucidum), que divide ambos ventrículos.

- Identificar el tronco cerebral como referencia anatómica.

- Realizar la medición en ventana transtemporal izquierda y derecha, aplicar la fórmula (A-B)/2, siendo A la distancia mayor en milímetros menos B la distancia menor en milímetros entre 2.

- Reportar la medición en milímetros y si la desviación es hacia la izquierda o derecha.

- Si la distancia mayor está a la derecha, la línea media está desviada a la izquierda y visceversa.

Método en pacientes craniectomizados

Insonar directamente sobre el defecto óseo utilizando el desplazamiento del septum pellucidum en relación con la hoz hiperecogénica del cerebro, siendo esta última empleada como referencia de la línea media. 
ANEXO 3

RESULTADOS

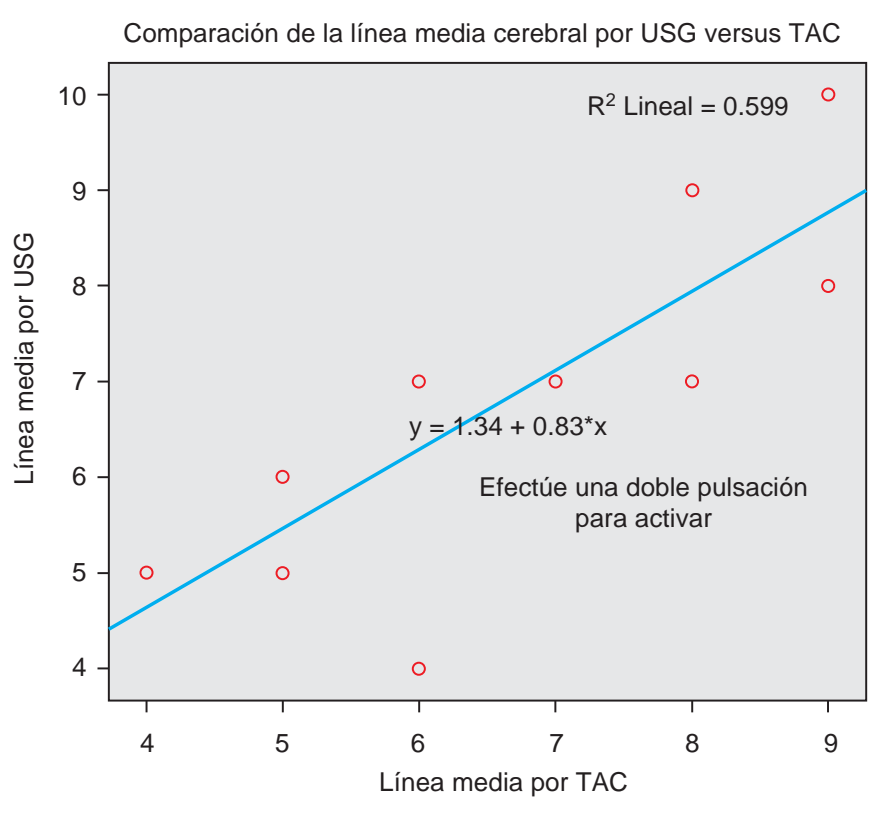

Estadísticos descriptivos

\begin{tabular}{lccc}
\hline & Media & Desviación estándar & $\mathrm{n}$ \\
\hline Línea media por USG & 6.81 & 1.601 & 22 \\
Línea media por TAC & 6.63 & 1.500 & 22 \\
\hline
\end{tabular}

Correlaciones

\begin{tabular}{lcc}
\hline & $\begin{array}{c}\text { Línea media } \\
\text { por USG }\end{array}$ & $\begin{array}{c}\text { Línea media } \\
\text { por TAC }\end{array}$ \\
\hline Línea media por USG & & \\
$\quad$ Correlación de Pearson & 1.000 & 0.774 \\
Sig. (bilateral) & & 0.000 \\
Suma de cuadros y productos vectoriales & 38.438 & 27.875 \\
Covarianza & 2.563 & 1.858 \\
$n$ & 22.000 & 22.000 \\
Línea media por TAC & & \\
Correlación de Pearson & 0.774 & 1.000 \\
Sig. (bilateral) & 0.000 & \\
Suma de cuadros y productos vectoriales & 27.875 & 33.750 \\
Covarianza & 1.858 & 2.250 \\
$\mathrm{n}$ & 22.000 & 22.000 \\
\hline
\end{tabular}

